\title{
Metformin Use is Associated with A Lower Incidence of Hospitalization for Atrial Fibrillation in Patients with Type 2 Diabetes Mellitus
}

Chin-Hsiao Tseng ( $\sim$ ccktsh@ms6.hinet.net)

National Taiwan University Hospital

Original investigation

Keywords: atrial fibrillation, diabetes mellitus, metformin, Taiwan

Posted Date: July 27th, 2020

DOI: https://doi.org/10.21203/rs.3.rs-44224/v1

License: (c) (i) This work is licensed under a Creative Commons Attribution 4.0 International License. Read Full License 


\section{Abstract}

Background: The effect of metformin on the risk of atrial fibrillation (AF) requires confirmation. This study compared the incidence of hospitalization for $\mathrm{AF}$ in ever and never users of metformin.

Methods: Patients with newly diagnosed type 2 diabetes mellitus during 1999-2005 were enrolled from Taiwan's National Health Insurance database. Analyses were conducted in both an unmatched cohort of 173398 ever users and 21666 never users and in a propensity score-matched cohort of 21647 pairs of ever and never users. They were free from a diagnosis of AF before January 1, 2006 and were followed up until December 31,2011 . Hazard ratios were estimated by Cox regression incorporated with the inverse probability of treatment weighting using the propensity score.

Results: A total of 303 ever users and 86 never users in the unmatched cohort and 50 ever users and 86 never users in the matched cohort developed hospitalization for AF during follow-up. The respective incidence rates were 37.72 and 92.45 per 100,000 person-years in the unmatched cohort and were 50.71 and 92.52 per 100,000 person-years in the matched cohort. The hazard ratio for ever versus never users was 0.405 ( $95 \%$ confidence interval: $0.319-0.515)$ in the unmatched cohort and 0.548 (0.387-0.777) in the matched cohort. Hazard ratios for the tertiles of cumulative duration of metformin therapy versus never users showed a dose-response effect. The findings were consistent in sensitivity analyses.

Conclusion: Metformin use is associated with a lower risk of hospitalization for AF in patients with type 2 diabetes mellitus.

\section{Introduction}

Atrial fibrillation (AF) is a common arrhythmia that can lead to increased hospitalization, stroke, or even life-threatening thromboembolic events [1, 2]. Risk factors of AF may include aging, male sex, smoking, alcohol consumption, diabetes mellitus, hypertension, obesity, thyroid dysfunction, obstructive sleep apnea, high-level physical training, left ventricular dysfunction, valvular disease, myocardial infarction and heart failure [2-4]. The estimated lifetime risk of AF is $22-26 \%$ and diabetes patients suffer from a $1.4-1.6$ fold higher risk [2, 3]. While compared to diabetes patients without AF, diabetes patients with AF have a $61 \%$ higher risk of total mortality, $77 \%$ higher risk of cardiovascular death and $68 \%$ higher risk of heart failure [5].

Metformin, now a first-line oral antidiabetic drug recommended for the treatment of type 2 diabetes mellitus, exerts an insulin sensitizing effect [6] and may have anti-inflammatory, anti-aging, anti-cancer and even anti-microbial effects [7-10]. Two previous pharmacoepidemiological studies conducted in Taiwan that used the nationwide reimbursement database of the National Health Insurance (NHI) showed that patients with type 2 diabetes mellitus treated with metformin might have a $20 \%$ lower risk of AF $[11,12]$. However, these earlier studies suffered from some methodological limitations.

There are several methodological limitations in the study by Chang et al. [11]. First, the metformin users and non-users enrolled were highly imbalanced in the use of statin with a significantly higher rates of statin use among metformin users. Because statin may reduce the incidence of AF by $40 \%-50 \%[13,14]$, the lower risk of AF among incident users of metformin could be potentially ascribed to the effect of statin which had been in use for a certain period before metformin was initiated. Second, the significantly higher prevalence rates of important risk factors of AF among non-users of metformin, such as male patients, older age, congestive heart failure, chronic kidney disease, asthma, hyperthyroidism, myocardial infarction, ischemic stroke and peripheral arterial disease, might have exerted residual confounding effects even though some of them were considered in the adjusted model. Third, imbalance in the use of other antidiabetic drugs between metformin users and non-users was highly probable and this might have led to a biased result. The investigators excluded, at the beginning of the study, users of other antidiabetic drugs and compared the incident cases of AF between metformin users and non-users over time by using a time-dependent approach. In this sense metformin non-users represented a highly selective group of patients who had a diagnosis of diabetes mellitus but had not been treated with any other antidiabetic drugs at the beginning of the study. On the other hand, non-users of metformin at the beginning of the study would only represent those with a diagnosis but without taking any antidiabetic drug. During subsequent years of follow-up, non-users of metformin might have been prescribed metformin or other antidiabetic drugs. Once metformin was prescribed in the subsequent years, the patients would be reclassified as metformin users. It was expected that insulin or insulin-secreting drugs, which could cause hypoglycemia and thus AF, would have been prescribed to metformin non-users for the purpose of glycemic control in the subsequent years of follow-up. Therefore, the proportions of the use of other antidiabetic drugs that could cause hypoglycemia and AF would be much higher among metformin non-users during any subsequent year of followup. Finally, this study did not consider the requirement of a sufficient induction time and did not address the potential bias due to immortal time. Therefore, selection bias, confounding by indication and immortal time bias could not be excluded in this study.

In the study by Liou et al., a nested case-control design was used by including 11528 diabetes patients without AF and 2882 diabetes patients with AF [12]. The investigators cross-sectionally estimated the odds ratios of AF for metformin users versus metformin non-users [12]. The crosssectional design did not assure a temporal correctness of a cause (metformin use) that should happen before an effect (AF incidence). It is interesting that although a matching algorithm was used, the enrolled cases (AF group) and controls (non-AF group) were not well matched in most of the important confounders. For example, the AF group was characterized by significantly higher prevalent rates of potential risk factors of AF including hypertension, congestive heart failure, chronic kidney disease, acute myocardial infarction and ischemic stroke than the non-AF group. 
Furthermore, the enrolled AF group was characterized by significantly higher prevalence rates of the use of insulin, sulfonylurea, glinide, alphaglucosidase inhibitors and dipeptidyl-peptidase 4 inhibitors [12]. Because insulin and insulin-secreting drugs may induce hypoglycemia which would lead to AF, residual confounding from other antidiabetic drugs could not be excluded. Therefore, this study could not clarify the temporal correctness of a cause-effect relationship and might have suffered from selection bias, prevalent user bias, immortal time bias and confounding by indication.

Some other common limitations found in these two earlier studies $[11,12]$ included a lack of investigating a dose-response effect and an ignorance of the potential impact of the regularity of metformin use. Additionally, the diagnosis of AF was made mainly at outpatient settings in these two studies, which was prone to be misclassified than a diagnosis made at the discharge of a hospitalization that is always supported by laboratory tests performed during admission.

A recent study conducted in the USA showed an increasing trend of emergency department visits and hospitalization for AF (HAF) from 2007 to 2014 in a nationwide level and called for a "need for widespread implementation of effective strategies aimed at improving the management of patients with AF to reduce hospital admissions and the economic burden of AF" [15]. Because metformin is considered the first-line antidiabetic drug for patients with type 2 diabetes mellitus, whether the use of metformin can prevent the potentially fatal disease of AF in these high-risk diabetes patients is of immense clinical importance. The purpose of the present study was to clarify whether metformin could reduce the incidence of AF made at the discharge of a hospitalization by using the Taiwan's NHI reimbursement database. To examine the consistency of the findings, analyses were conducted both in an unmatched cohort and a propensity score (PS)-matched cohort of patients with type 2 diabetes mellitus followed by sensitivity analyses after excluding patients with irregular refill of metformin and after excluding patients who happened to be treated with incretinbased therapies during follow-up. The potential methodological limitations seen in the previous studies [11, 12] were also addressed.

\section{Materials And Methods}

The NHI has been implemented in Taiwan since March 1995. It is a unique and universal health care system with a high coverage rate of $>99 \%$ of the Taiwan's population. The Bureau of the NHI has contracts with all hospitals and with $93 \%$ of all medical settings. The reimbursement database of the NHI kept computerized records of disease diagnoses, medication prescriptions and clinical procedures and can be used for academic research if approved after ethics review. The present study was approved number 99274 by the Ethics Committee of the National Health Research Institutes. According to local regulations, the National Health Research Institutes de-identified the individuals in the database for the protection of privacy and the Ethics Committee approved the analyses of the database without the requirement of obtaining informed consent from the participants.

During the study period, the International Classification of Diseases, Ninth Revision, Clinical Modification (ICD-9-CM) was used for disease diagnoses; and diabetes mellitus was coded as 250.XX and AF as 427.31.

Detailed description of the database has been reported in a previously published paper [16]. The unmatched cohort and the PS-matched cohort were enrolled according to the procedures shown in Fig. 1. At first, 423949 patients who were newly diagnosed of diabetes mellitus during $1999-2005$ in the outpatient clinics and had been prescribed antidiabetic drugs for two or more times were identified. The following ineligible patients were then excluded: 1) metformin ever users who had been treated with other antidiabetic drugs before metformin was initiated ( $n=183837)$; 2$)$ type 1 diabetes mellitus ( $n=2062), 3$ ) patients who died before the start of follow-up $(n=65) ; 4)$ missing data $(n=358), 5)$ diagnosis of AF before the start of follow-up or within 6 months of diabetes diagnosis $(n=812), 6)$ diagnosis of any cancer before the start of follow-up or within 6 months of diabetes diagnosis $(n=26675)$, and 7 ) follow-up duration < 180 days ( $n=15076)$. As a result, 173398 ever users and 21666 never users of metformin were identified as the unmatched cohort. PS was created from all characteristics listed in Table 1 plus the date of enrollment by logistic regression. A cohort of PS-matched pairs of 21647 ever users and 21647 never users (the matched cohort) was then created from the unmatched cohort by matching the PS using the Greedy $8 \diamond 1$ digit match algorithm proposed by Parsons [17]. 
Table 1

Characteristics in never users and ever users of metformin in the unmatched and matched cohorts

\begin{tabular}{|c|c|c|c|c|c|c|c|c|c|c|}
\hline \multirow[t]{4}{*}{ Variable } & \multicolumn{5}{|c|}{ Unmatched cohort } & \multicolumn{5}{|c|}{ Matched cohort } \\
\hline & \multirow{2}{*}{\multicolumn{2}{|c|}{$\begin{array}{l}\text { Never users } \\
(n=21666)\end{array}$}} & \multicolumn{3}{|c|}{ Ever users } & \multirow{2}{*}{\multicolumn{2}{|c|}{$\begin{array}{l}\text { Never users } \\
(n=21647)\end{array}$}} & \multicolumn{3}{|c|}{ Ever users } \\
\hline & & & $(n=173$ & & Standardized & & & $(n=216$ & 17) & Standardized \\
\hline & $\mathrm{n}$ & $\%$ & $\mathrm{n}$ & $\%$ & & $\mathrm{n}$ & $\%$ & $\mathrm{n}$ & $\%$ & \\
\hline \multicolumn{11}{|l|}{ Demographic data } \\
\hline Age* (years) & 68.77 & 13.25 & 64.23 & 11.98 & -41.28 & 68.76 & 13.25 & 68.69 & 12.13 & 0.15 \\
\hline Sex (men) & 11793 & 54.43 & 91570 & 52.81 & -3.51 & 11781 & 54.42 & 11902 & 54.98 & 1.09 \\
\hline \multicolumn{11}{|l|}{ Occupation } \\
\hline I & 7825 & 36.12 & 64789 & 37.36 & & 7818 & 36.12 & 7989 & 36.91 & \\
\hline II & 3646 & 16.83 & 36710 & 21.17 & 12.59 & 3645 & 16.84 & 3542 & 16.36 & -1.39 \\
\hline III & 5216 & 24.07 & 39817 & 22.96 & -2.67 & 5212 & 24.08 & 5152 & 23.80 & -0.46 \\
\hline IV & 4979 & 22.98 & 32082 & 18.50 & -12.94 & 4972 & 22.97 & 4964 & 22.93 & -0.01 \\
\hline \multicolumn{11}{|l|}{ Living region } \\
\hline Taipei & 7328 & 33.82 & 54416 & 31.38 & & 7318 & 33.81 & 7358 & 33.99 & \\
\hline Northern & 2310 & 10.66 & 20016 & 11.54 & 2.90 & 2308 & 10.66 & 2312 & 10.68 & 0.02 \\
\hline Central & 3769 & 17.40 & 31678 & 18.27 & 2.12 & 3767 & 17.40 & 3757 & 17.36 & -0.17 \\
\hline Southern & 3729 & 17.21 & 29840 & 17.21 & 0.39 & 3725 & 17.21 & 3684 & 17.02 & -0.28 \\
\hline Kao-Ping and Eastern & 4530 & 20.91 & 37448 & 21.60 & 2.52 & 4529 & 20.92 & 4536 & 20.95 & 0.22 \\
\hline \multicolumn{11}{|l|}{ Major comorbidities } \\
\hline Hypertension & 18476 & 85.28 & 144909 & 83.57 & -5.92 & 18461 & 85.28 & 18453 & 85.25 & 0.13 \\
\hline Dyslipidemia & 15127 & 69.82 & 141918 & 81.85 & 32.52 & 15123 & 69.86 & 15238 & 70.39 & 1.18 \\
\hline Obesity & 518 & 2.39 & 7364 & 4.25 & 11.03 & 518 & 2.39 & 524 & 2.42 & 0.11 \\
\hline \multicolumn{11}{|l|}{$\begin{array}{l}\text { Diabetes-related } \\
\text { complications }\end{array}$} \\
\hline Nephropathy & 8376 & 38.66 & 50614 & 29.19 & -24.68 & 8362 & 38.63 & 8264 & 38.18 & -1.33 \\
\hline Eye diseases & 3741 & 17.27 & 55119 & 31.79 & 35.85 & 3740 & 17.28 & 3657 & 16.89 & -1.43 \\
\hline Stroke & 8870 & 40.94 & 58105 & 33.51 & -18.48 & 8861 & 40.93 & 8860 & 40.93 & 0.06 \\
\hline $\begin{array}{l}\text { Ischemic heart } \\
\text { disease }\end{array}$ & 11689 & 53.95 & 84499 & 48.73 & -12.53 & 11679 & 53.95 & 11706 & 54.08 & 0.36 \\
\hline $\begin{array}{l}\text { Peripheral arterial } \\
\text { disease }\end{array}$ & 5678 & 26.21 & 47465 & 27.37 & 2.10 & 5672 & 26.20 & 5763 & 26.62 & 0.78 \\
\hline \multicolumn{11}{|l|}{ Antidiabetic drugs } \\
\hline Insulin & 1891 & 8.73 & 3953 & 2.28 & -34.85 & 1877 & 8.67 & 1649 & 7.62 & -5.81 \\
\hline Sulfonylurea & 15450 & 71.31 & 123550 & 71.25 & 8.93 & 15447 & 71.36 & 16073 & 74.25 & 5.93 \\
\hline Meglitinide & 1958 & 9.04 & 6930 & 4.00 & -22.82 & 1952 & 9.02 & 1924 & 8.89 & -0.71 \\
\hline Acarbose & 2443 & 11.28 & 9223 & 5.32 & -20.85 & 2438 & 11.26 & 2558 & 11.82 & -0.13 \\
\hline Rosiglitazone & 618 & 2.85 & 8158 & 4.70 & 10.80 & 618 & 2.85 & 618 & 2.85 & -0.79 \\
\hline Pioglitazone & 510 & 2.35 & 4356 & 2.51 & 2.42 & 509 & 2.35 & 532 & 2.46 & -0.17 \\
\hline
\end{tabular}

*Age is expressed as mean and standard deviation

Refer to "Materials and Methods" for the classification of occupation 


\begin{tabular}{|c|c|c|c|c|c|c|c|c|c|}
\hline \multirow[t]{4}{*}{ Variable } & \multicolumn{4}{|c|}{ Unmatched cohort } & \multicolumn{5}{|c|}{ Matched cohort } \\
\hline & \multirow{2}{*}{$\begin{array}{l}\text { Never users } \\
(n=21666)\end{array}$} & \multicolumn{3}{|c|}{ Ever users } & \multicolumn{2}{|c|}{ Never users } & \multicolumn{3}{|c|}{ Ever users } \\
\hline & & & & $\begin{array}{l}\text { Standardized } \\
\text { difference }\end{array}$ & & & & & $\begin{array}{l}\text { Standardized } \\
\text { difference }\end{array}$ \\
\hline & $\%$ & $\mathrm{n}$ & $\%$ & & $\mathrm{n}$ & $\%$ & $\mathrm{n}$ & $\%$ & \\
\hline
\end{tabular}

Commonly encountered comorbidities and potential risk factors of atrial fibrillation

\begin{tabular}{|c|c|c|c|c|c|c|c|c|c|c|}
\hline $\begin{array}{l}\text { Chronic obstructive } \\
\text { pulmonary disease }\end{array}$ & 12054 & 55.64 & 89210 & 51.45 & -10.90 & 12039 & 55.62 & 11904 & 54.99 & -1.12 \\
\hline Tobacco abuse & 507 & 2.34 & 6439 & 3.71 & 8.81 & 507 & 2.34 & 473 & 2.19 & -1.07 \\
\hline $\begin{array}{l}\text { Alcohol-related } \\
\text { diagnoses }\end{array}$ & 1406 & 6.49 & 11443 & 6.60 & -0.11 & 1405 & 6.49 & 1338 & 6.18 & -1.51 \\
\hline Cancer & 2254 & 10.40 & 13711 & 7.91 & -9.64 & 2249 & 10.39 & 2260 & 10.44 & 0.20 \\
\hline Heart failure & 6237 & 28.79 & 35109 & 20.25 & -24.13 & 6229 & 28.78 & 6145 & 28.39 & -0.96 \\
\hline Gout & 8921 & 41.18 & 63552 & 36.65 & -10.62 & 8915 & 41.18 & 8930 & 41.25 & 0.32 \\
\hline Hyperthyroidism & 1030 & 4.75 & 8637 & 4.98 & 1.31 & 1029 & 4.75 & 1046 & 4.83 & 0.42 \\
\hline $\begin{array}{l}\text { Sleep apnea } \\
\text { syndrome }\end{array}$ & 413 & 1.91 & 3378 & 1.95 & 0.40 & 412 & 1.90 & 391 & 1.81 & -0.69 \\
\hline \multicolumn{11}{|l|}{$\begin{array}{l}\text { Commonly used } \\
\text { medications in } \\
\text { diabetes patients }\end{array}$} \\
\hline $\begin{array}{l}\text { Angiotensin- } \\
\text { converting enzyme } \\
\text { inhibitors/angiotensin } \\
\text { receptor blockers }\end{array}$ & 15959 & 73.66 & 130207 & 75.09 & 2.83 & 15946 & 73.66 & 15903 & 73.47 & -0.37 \\
\hline $\begin{array}{l}\text { Calcium channel } \\
\text { blockers }\end{array}$ & 14776 & 68.20 & 109003 & 62.86 & -12.81 & 14760 & 68.18 & 14754 & 68.16 & 0.21 \\
\hline Beta-blockers & 15951 & 73.62 & 119761 & 69.07 & -11.13 & 15936 & 73.62 & 15979 & 73.82 & 0.64 \\
\hline Statins & 11326 & 52.28 & 112600 & 64.94 & 29.01 & 11323 & 52.31 & 11421 & 52.76 & 0.76 \\
\hline Fibrates & 7090 & 32.72 & 73563 & 42.42 & 22.44 & 7090 & 32.75 & 7126 & 32.92 & 0.29 \\
\hline Aspirin & 13582 & 62.69 & 111115 & 64.08 & 2.06 & 13572 & 62.70 & 13602 & 62.84 & 0.44 \\
\hline
\end{tabular}

*Age is expressed as mean and standard deviation

Refer to "Materials and Methods" for the classification of occupation

The start of follow up was set on January 1, 2006 and all comorbidities and covariates were determined as a status/diagnosis at any time before the start of follow-up. Potential confounders included: 1) demographic data: age, sex, occupation and living region; 2) major comorbidities: hypertension (401-405), dyslipidemia (272.0-272.4) and obesity (278); 3) diabetes-related complications: nephropathy (580-589), eye diseases (250.5: diabetes with ophthalmic manifestations, 362.0: diabetic retinopathy, 369: blindness and low vision, 366.41: diabetic cataract, and 365.44: glaucoma associated with systemic syndromes), stroke (430-438), ischemic heart disease (410-414) and peripheral arterial disease (250.7, 785.4, 443.81 and 440-448); 4) antidiabetic drugs: insulin, sulfonylurea, meglitinide, acarbose, rosiglitazone and pioglitazone; 5) commonly encountered comorbidities and potential risk factors of AF: chronic obstructive pulmonary disease (a surrogate for smoking, 490-496), tobacco abuse (305.1, 
649.0 and 989.84), alcohol-related diagnoses (291, 303, 535.3, 571.0-571.3 and 980.0), cancer (140-208), heart failure (398.91, 402.11, 402.91, $404.11,404.13,404.91,404.93$ and 428), gout (274), hyperthyroidism (242) and sleep apnea syndrome (327.2, 780.51, 780.53, 780.57); and 6) commonly used medications in diabetes patients: angiotensin-converting enzyme inhibitors/angiotensin receptor blockers, calcium channel blockers, beta-blockers, statins, fibrates and aspirin. The accuracy of disease diagnoses in the NHI database has been studied previously. Agreements between claim data and medical records are moderate to substantial, with Kappa values range from 0.55 to 0.86 [18].

The classifications of living region and occupation were detailed elsewhere [19]. In brief, the living region was classified as Taipei, Northern, Central, Southern, and Kao-Ping/Eastern. Occupation was classified as class I (civil servants, teachers, employees of governmental or private businesses, professionals and technicians), class II (people without a specific employer, self-employed people or seamen), class III (farmers or fishermen) and class IV (low-income families supported by social welfare, or veterans).

Standardized difference was calculated according to the methods proposed by Austin and Stuart for each covariate as a test of balance diagnostic [20]. A value of $>10 \%$ is used as a cutoff for potential confounding from the variable.

Cumulative duration of metformin therapy in months was calculated and its tertiles were used for dose-response analyses. Incidence density of HAF was calculated for never users, ever users and users categorized according to the tertiles of cumulative duration of metformin therapy. The numerator of the incidence was the case number of newly diagnosed HAF as a primary diagnosis at the discharge of a hospitalization observed during follow-up. The denominator expressed in person-years was the follow-up time since January 1, 2006 until December 31, 2011, when a new diagnosis of HAF was made, or on the date of death or the last reimbursement record, whichever occurred first.

Kaplan-Meier curves for HAF-free probability were plotted for never users and ever users of metformin and for never users and users categorized according to the tertiles of cumulative duration of metformin therapy. Logrank test was used to test the significance in different subgroups of metformin exposure.

In main analyses, hazard ratios and their 95\% confidence intervals were estimated by Cox proportional hazards model incorporated with the inverse probability of treatment weighting (IPTW) using the PS. This method was proposed by Austin for reducing the potential confounding from the differences in characteristics [21]. Models were created for ever users versus never users and for users in each tertile of cumulative duration of metformin therapy in comparison to never users.

For sensitivity analyses, the following models were created in the unmatched cohort by using both the IPTW method and the traditional Cox regression: 1) Excluding patients with irregular refill of metformin, based on two consecutive prescriptions of metformin spanning a period of $>4$ months (the Bureau of $\mathrm{NHI}$ allows a maximum of 3 months at each time of drug prescription for chronic diseases and these patients with delayed refill of metformin for more than one month after a previous 3-month prescription might represent those with poor adherence); and 2) Excluding patients who happened to be treated with incretin-based therapies, i.e., dipeptidyl peptidase 4 inhibitors and glucagon-like peptide 1 receptor agonists, during follow-up (incretin-based therapies were not reimbursed by the Bureau of NHI in Taiwan until after 2009 and this analysis was aimed at excluding their potential impact).

Analyses were conducted using SAS statistical software, version 9.4 (SAS Institute, Cary, NC). $P<0.05$ was considered statistically significant.

\section{Results}

Table 1 shows the characteristics in never users and ever users of metformin in the unmatched cohort and the matched cohort, respectively. Before matching, ever users and never users showed imbalance in the distribution of many covariates with standardized difference $>10 \%$. However, after matching, the two groups were well balanced in all covariates and none of the variables had a value of standardized difference $>10 \%$.

Figures 2 shows the Kaplan-Meier curves comparing HAF-free probability in never users and ever users of metformin (Fig. 2A) and in never users and users in each of the tertiles of cumulative duration of metformin therapy (Fig. 2B). The logrank test supported significant differences among the various subgroups of metformin exposure.

Table 2 shows the incidence of HAF and the hazard ratios by metformin exposure in the unmatched cohort and the matched cohort in the main analyses, respectively. A significantly lower risk of HAF in metformin ever users could be demonstrated by the overall hazard ratios and the tertile analysis supported a dose-response relationship. 
Table 2

Incidence of hospitalization for atrial fibrillation and hazard ratios by metformin exposure in the main analyses

\begin{tabular}{|c|c|c|c|c|c|c|c|}
\hline \multirow[t]{2}{*}{ Cohort/Metformin use } & \multirow[t]{2}{*}{$n$} & \multirow[t]{2}{*}{$N$} & \multirow[t]{2}{*}{ Person-year } & \multirow{2}{*}{$\begin{array}{l}\text { Incidence rate } \\
\text { (per } 100,000 \text { person-years) }\end{array}$} & \multicolumn{3}{|c|}{ IPTW model } \\
\hline & & & & & HR & $95 \% \mathrm{Cl}$ & $P$ value \\
\hline \multicolumn{8}{|l|}{ Unmatched cohort } \\
\hline Never users & 86 & 21666 & 93021.27 & 92.45 & 1.000 & & \\
\hline Ever users & 303 & 173398 & 803329.81 & 37.72 & 0.405 & $(0.319-0.515)$ & $<0.0001$ \\
\hline \multicolumn{8}{|c|}{ Tertiles of cumulative duration of metformin therapy (months) } \\
\hline Never users & 86 & 21666 & 93021.27 & 92.45 & 1.000 & & \\
\hline$<25.9$ & 121 & 57224 & 194246.52 & 62.29 & 0.664 & $(0.502-0.878)$ & 0.0041 \\
\hline $25.9-57.0$ & 107 & 57143 & 274026.03 & 39.05 & 0.419 & $(0.316-0.557)$ & $<0.0001$ \\
\hline$>57.0$ & 75 & 59031 & 335057.26 & 22.38 & 0.230 & $(0.168-0.314)$ & $<0.0001$ \\
\hline \multicolumn{8}{|l|}{ Matched cohort } \\
\hline Never users & 86 & 21647 & 92948.36 & 92.52 & 1.000 & & \\
\hline Ever users & 50 & 21647 & 98602.63 & 50.71 & 0.548 & $(0.387-0.777)$ & 0.0007 \\
\hline \multicolumn{8}{|c|}{ Tertiles of cumulative duration of metformin therapy (months) } \\
\hline Never users & 86 & 21647 & 92948.36 & 92.52 & 1.000 & & \\
\hline$<25.1$ & 19 & 7142 & 23685.13 & 80.22 & 0.858 & $(0.520-1.414)$ & 0.5471 \\
\hline $25.1-57.1$ & 16 & 7141 & 33527.36 & 47.72 & 0.518 & $(0.304-0.883)$ & 0.0156 \\
\hline$>57.1$ & 15 & 7364 & 41390.14 & 36.24 & 0.393 & $(0.227-0.681)$ & 0.0009 \\
\hline \multicolumn{8}{|c|}{$n$ : incident case number of hospitalization for atrial fibrillation, $N$ : case number followed } \\
\hline \multicolumn{8}{|c|}{ IPTW: inverse probability of treatment weighting } \\
\hline HR: hazard ratio, $\mathrm{Cl}$ : cor & dence & terval & & & & & \\
\hline
\end{tabular}

Table 3 shows the findings in the sensitivity analyses. All analyses supported a lower risk of HAF associated with metformin use in either the IPTW models or the traditional Cox regression models. 
Table 3

Sensitivity analyses

\begin{tabular}{|c|c|c|c|c|c|c|c|c|}
\hline \multirow[b]{2}{*}{ Model/metformin use } & \multicolumn{6}{|c|}{ IPTW model } & \multicolumn{2}{|c|}{ Traditional Cox model } \\
\hline & $n$ & $N$ & $\mathrm{HR}$ & $95 \% \mathrm{Cl}$ & $P$ value & $\mathrm{HR}$ & $95 \% \mathrm{Cl}$ & $P$ value \\
\hline \multicolumn{9}{|c|}{ Excluding two consecutive prescriptions of metformin spanning more than 4 months } \\
\hline Never users & 86 & 21666 & 1.000 & & & 1.000 & & \\
\hline Ever users & 93 & 59513 & 0.393 & $(0.293-0.527)$ & $<0.0001$ & 0.556 & $(0.405-0.763)$ & 0.0003 \\
\hline
\end{tabular}

Tertiles of cumulative duration of metformin therapy (months)

\begin{tabular}{lllllllll} 
Never users & 86 & 21666 & 1.000 & & \multicolumn{3}{c}{1.000} & \\
\hline$<25.2$ & 35 & 20075 & 0.708 & $(0.474-1.058)$ & 0.0922 & 1.166 & $(0.748-1.817)$ & 0.4983 \\
\hline $25.2-56.4$ & 27 & 16197 & 0.415 & $(0.269-0.640)$ & $<0.0001$ & 0.626 & $(0.398-0.982)$ & 0.0416 \\
$>56.4$ & 31 & 23241 & 0.245 & $(0.162-0.370)$ & $<0.0001$ & 0.332 & $(0.215-0.513)$ & $<0.0001$
\end{tabular}

Excluding two consecutive prescriptions of metformin spanning more than 6 months

$\begin{array}{llllllllll}\text { Never users } & 86 & 21666 & 1.000 & & & & & \\ \text { Ever users } & 125 & 78077 & 0.000 & (0.295-0.510) & <0.0001 & 0.577 & (0.429-0.777) & 0.0003\end{array}$

Tertiles of cumulative duration of metformin therapy (months)

\begin{tabular}{lllllllll} 
Never users & 86 & 21666 & 1.000 & & \multicolumn{3}{c}{1.000} & \\
\hline$<25.2$ & 44 & 23888 & 0.718 & $(0.496-1.041)$ & 0.0808 & 1.264 & $(0.840-1.901)$ & 0.2616 \\
\hline $25.2-56.4$ & 35 & 22271 & 0.375 & $(0.253-0.556)$ & $<0.0001$ & 0.590 & $(0.392-0.889)$ & 0.0116 \\
$\geq 56.4$ & 46 & 31918 & 0.262 & $(0.183-0.376)$ & $<0.0001$ & 0.373 & $(0.254-0.546)$ & $<0.0001$
\end{tabular}

Excluding patients treated with incretin-based therapies during follow-up

\begin{tabular}{lllllllll} 
Never users & 84 & 20402 & 1.000 & \multicolumn{3}{c}{1.000} \\
Ever users & 269 & 134549 & 0.458 & $(0.359-0.585)$ & $<0.0001$ & 0.640 & $(0.493-0.831)$ & 0.0008
\end{tabular}

Tertiles of cumulative duration of metformin therapy (months)

\begin{tabular}{lccccccccc} 
Never users & 84 & 20402 & 1.000 & & \multicolumn{3}{c}{1.000} & & \\
\hline$<25.2$ & 115 & 48775 & 0.713 & $(0.536-0.947)$ & 0.0196 & 1.143 & $(0.842-1.552)$ & 0.3904 \\
\hline $25.2-56.4$ & 94 & 44083 & 0.465 & $(0.347-0.625)$ & $<0.0001$ & 0.666 & $(0.489-0.905)$ & 0.0095 \\
\hline$>56.4$ & 60 & 41691 & 0.258 & $(0.185-0.361)$ & $<0.0001$ & 0.346 & $(0.245-0.489)$ & $<0.0001$
\end{tabular}

$n$ : incident case number of hospitalization for atrial fibrillation, $N$ : case number followed

IPTW: inverse probability of treatment weighting

HR: hazard ratio, Cl: confidence interval

\section{Discussion}

This study confirmed a lower risk of HAF diagnosed at the discharge of a hospitalization in patients with type 2 diabetes mellitus who had been treated with metformin (Tables 2 and 3). A dose-response pattern was seen and the beneficial effect was especially significant when metformin had been used for more than 2 years in either the main analyses (Table 2) or the sensitivity analyses (Table 3).

It is worthy to note that, by using the diagnostic code mainly identified at the outpatient clinics, the hazard ratio of 0.81 (95\% confidence interval 0.76-0.86) estimated by Chang et al. [11] and the odds ratio of 0.81 (95\% confidence interval 0.71-0.93) estimated by Liou et al. [12] showed a less magnitude of protection than what we have seen in the present study (Tables 2 and 3 ). This could be because that the diagnostic code of AF labelled at the outpatient clinics was not as accurate as the diagnosis made during hospitalization. The misclassification made at outpatient clinics was probably nondifferential and thus biased the hazard ratios toward the null [22]. It is true that when we used a less stringent definition of AF by including the diagnostic code identified either at the outpatient clinics or during hospitalization in secondary analyses, the estimated hazard ratios did move toward the null (overall hazard ratio for the matched cohort: $0.668,95 \%$ confidence interval: $0.603-0.741$ ), indicating a prone to nondifferential misclassification by using the diagnosis labelled at the outpatient clinics. However, despite the variation in the estimated risk ratios, 
all studies consistently supported a protection of metformin against AF by using different study designs and different statistical analyses and by defining AF either as a diagnosis made at the outpatient clinics or made at the discharge of a hospitalization.

The higher risk of AF in patients with type 2 diabetes mellitus is assumed to be related to metabolic syndrome and the pathogenesis may involve insulin resistance, hypertension, greater glycemic excursion, hypoglycemia, myocardial steatosis, endothelial dysfunction, inflammation and left atrial dilatation and fibrosis [3]. These can lead to electrical and structural remodeling and AF [3]. Therefore, even though the mechanisms of a reduced risk of $\mathrm{AF}$ associated with metformin use are not yet elucidated, the biological actions of metformin targeting the pathophysiology of the development of AF might have contributed to such a clinical benefit.

Metformin improves insulin resistance by increasing the expression of insulin receptor and activation of tyrosine kinase [23]. In the absence of coadministration of insulin or insulin-stimulating drugs, metformin per se rarely induce hypoglycemia. On the other hand, microvascular dysfunction and relative ischemia with reduced oxygen/nutrient delivery and/or increased energy demand in the heart may lead to metabolic stress and induce AF [1]. Through the activation of 5'-adenosine monophosphate-activated protein kinase, metformin promotes fatty acid oxidation, increases ketone body metabolism, reduces lipid accumulation and induces the expression of glucose transporter in cardiomyocytes, thus facilitates more efficient energy use with reduction of metabolic stress [1,24]. Studies also suggested that metformin may reduce AF by alleviating the dysfunction of epicardial adipose tissue [25]. In an in vitro study, metformin reduces the production of reactive oxygen species and myolysis during tachypacing cell culture of atrial myocytes [11].

Pro-fibrotic biomarkers such as interleukin-6, transforming growth factor-beta one, matrix metalloproteinase-9 and tissue inhibitor of metalloproteinase-1 are important biomarkers of atrial remodeling in AF [1, 26]. Interestingly, metformin inhibits the signaling pathways of transforming growth factor-beta one [27]. Taken together, the mechanisms of a reduced risk of AF associated with metformin use may be multifactorial and are related to a reduction of insulin resistance and metabolic stress, inhibition of inflammation and alleviation of cardiac fibrosis.

The findings of the present study have some clinical implications. First, because diabetes patients have a higher risk of AF [2-4] which may contribute to the significantly higher risk of thromboembolic events and mortality [5], the reduced risk of AF associated with metformin use provided a good rationale for the recommendation of metformin as the first-line treatment for glucose lowering in patients with type 2 diabetes mellitus. Second, it would be a good strategy to continue the use of metformin when the addition of other antidiabetic drugs is required for better control of hyperglycemia because its protection against AF may or may not be directly related to glucose lowering and such a protection was observed mainly after two years of its use (Tables 2 and 3). Furthermore, the dose-response pattern (Tables 2 and 3 ) not only implicates a cause-effect relationship but also provides a good rationale for its continuous use when other antidiabetic drugs are added for the improvement of hyperglycemia. Third, the consistent findings of a protective role of metformin on AF in the diabetes patients might provide rationale for more vigorous investigation of its usefulness in non-diabetes people who are at a high risk of developing AF.

Pharmacoepidemiological studies using administrative databases to evaluate long-term safety or beneficial/adverse effects of medications have become popular in recent years. These studies are most useful for outcomes with low incidence or when randomized controlled trials are not feasible. However, some methodology limitations should be carefully addressed for not getting a biased result. Methodological limitations such as selection bias, prevalent user bias, immortal time bias and confounding by indication as seen in the two previous studies [11, 12] have been carefully addressed in the present study during study design, patient enrollment and statistical analyses. Selection bias was avoided by using the nationwide database covering $>99 \%$ of the population and prevalent user bias was prevented by enrolling patients with new-onset diabetes and new users of metformin. The impacts or carryover effects of other antidiabetic drugs used before metformin was initiated were also excluded by enrolling only ever users of metformin who received metformin as the first antidiabetic drug (Fig. 1).

Immortal time refers to the follow-up period during which the outcome cannot happen. Immortal time bias can be introduced when treatment status and/or follow-up time are inappropriately assigned [28]. To prevent misclassification of non-diabetes people as diabetes patients, those with uncertain diabetes diagnosis have been excluded by enrolling only patients who had been prescribed antidiabetic drugs for two or more times (Fig. 1). Misclassification of the treatment status of metformin was not likely because the NHI is a universal health care system and the information of all prescriptions is available during the long period of follow-up. The immortal time during the period between diabetes diagnosis and the initiation of antidiabetic drugs and during the initial short follow-up period of $<180$ days were not included in the calculation of the follow-up personyears. Lastly, the immortal time during the waiting period between drug prescription and drug dispense when a patient is discharged from the hospital (as pointed out by Lévesque et al. [28]) is not a problem in Taiwan because the patient can get all discharge medications immediately from the hospital at the time of his/her discharge.

There are some additional strengths. First, recall bias resulting from self-reporting would not happen in the study because medical records were used. Second, although the detection rate of a disease might be affected by socioeconomic status of the patients in studies conducted in other countries, this was less likely in Taiwan because the drug cost-sharing in the NHI health care system is low and much expenses can be waived in veterans, in patients with low-income or when the patients receive prescription refills for chronic disease.

The consistency of the findings in both the unmatched cohort and the matched cohort (Table 2) and in the sensitivity analyses by using both the IPTW method and the traditional Cox regression (Table 3) suggested that the results were reproducible in different cohorts and by using different 
statistical models. The use of a PS-matched cohort (Table 1) and the estimation of hazard ratios by using the Cox proportional hazards model incorporated with IPTW (Tables 2 and 3) was aimed at reducing potential confounding by indication. The possibility of residual confounding from the covariates should be small, especially in the matched cohort for whom the values of standardized difference between ever and never users of metformin were $<10 \%$ for all covariates (Table 1 ).

Study limitations may include a lack of the availability of electrocardiograms for confirming the diagnosis of AF. Therefore, misclassification of AF could not be completely excluded. However, if the misclassifications were not differential in ever and never users of metformin, the hazard ratios would only have been underestimated [22]. To minimize the misclassification of AF, we used a more stringent definition of AF by including only those who were hospitalized for the management of AF. It is believed that the primary diagnosis labelled at hospital discharge should have been supported by laboratory information such as electrocardiograms or electrophysiological studies. Other limitations might include a lack of measurement data of some confounders like biochemical and hormonal data, anthropometric factors, lifestyle, physical activity, dietary pattern, cigarette smoking, alcohol drinking, family history and genetic parameters.

\section{Conclusion}

This study supports a lower risk of HAF in patients with type 2 diabetes mellitus who have been treated with metformin. However, additional prospective observational studies and/or clinical trials are necessary to confirm a cause-effect relationship. Because metformin does not cause hypoglycemia when used as monotherapy and it is inexpensive and safe, the usefulness of metformin as a protection against AF in high risk patients is worthy of more intensive investigation in both the diabetes patients and the non-diabetes people.

\section{Abbreviations}

AF: atrial fibrillation

HAF: hospitalization for atrial fibrillation

ICD-9-CM: International Classification of Diseases, Ninth Revision, Clinical Modification

IPTW: inverse probability of treatment weighting

NHI: National Health Insurance

PS: propensity score

\section{Declarations}

\section{Ethics approval and consent to participate}

This study used secondary data from the $\mathrm{NHI}$ reimbursement database according to the local regulations and received an approval number of 99274. No informed consent from the patients was required because personal information has been de-identified.

\section{Consent for publication}

Not applicable

\section{Availability of data and material}

Due to the local law restriction on the release of individualized data to the public for the protection of privacy, the data and study materials will not be made available to other researchers.

\section{Competing interests}

The author declares no conflicts of interest.

\section{Funding}

The study was supported by the Ministry of Science and Technology (MOST 107-2221-E-002-129-MY3) of Taiwan and the Yee Fong Charity Foundation. The funders had no role in study design, data collection and analysis, decision to publish, or preparation of the manuscript.

\section{Authors' contributions}

Tseng $\mathrm{CH}$ researched data and wrote manuscript. 


\section{Acknowledgements}

The study is based in part on data from the National Health Insurance Research Database provided by the Bureau of National Health Insurance, Department of Health and managed by National Health Research Institutes. The interpretation and conclusions contained herein do not represent those of Bureau of National Health Insurance, Department of Health or National Health Research Institutes.

\section{Authors' information (optional)}

Not applicable

\section{Conflicts of interest: None}

\section{References}

1. Harada M, Melka J, Sobue Y, Nattel S. Metabolic considerations in atrial fibrillation - Mechanistic insights and therapeutic opportunities. Circ J. 2017;81:1749-1757

2. Andrade J, Khairy P, Dobrev D, Nattel S. The clinical profile and pathophysiology of atrial fibrillation: relationships among clinical features, epidemiology, and mechanisms. Circ Res. 2014;114:1453-68.

3. Bell DSH, Goncalves E. Atrial fibrillation and type 2 diabetes: Prevalence, etiology, pathophysiology and effect of anti-diabetic therapies. Diabetes Obes Metab. 2019;21:210-217.

4. Boriani G, Proietti M. Atrial fibrillation prevention: an appraisal of current evidence. Heart. 2018;104:882-887.

5. Du X, Ninomiya T, de Galan B, Abadir E, Chalmers J, Pillai A, Woodward M, Cooper M, Harrap S, Hamet P, Poulter N, Lip GY, Patel A; ADVANCE Collaborative Group. Risks of cardiovascular events and effects of routine blood pressure lowering among patients with type 2 diabetes and atrial fibrillation: results of the ADVANCE study. Eur Heart J. 2009;30:1128-35.

6. Yang X, Xu Z, Zhang C, Cai Z, Zhang J. Metformin, beyond an insulin sensitizer, targeting heart and pancreatic $\beta$ cells. Biochim Biophys Acta. 2017;1863:1984-90.

7. Maniar K, Moideen A, Mittal A, Patil A, Chakrabarti A, Banerjee D. A story of metformin-butyrate synergism to control various pathological conditions as a consequence of gut microbiome modification: Genesis of a wonder drug? Pharmacol Res. 2017;117:103-128.

8. Tseng CH. Metformin and Helicobacter pylori infection in type 2 diabetes patients. Diabetes Care. 2018;41:e42-e43.

9. Tseng CH. Metformin decreases risk of tuberculosis infection in type 2 diabetes patients. J Clin Med. 2018 Sep 9;7(9).

10. Tseng $\mathrm{CH}$. Metformin and risk of chronic obstructive pulmonary disease in diabetes patients. Diabetes Metab. 2019;45:184-190.

11. Chang SH, Wu LS, Chiou MJ, Liu JR, Yu KH, Kuo CF, Wen MS, Chen WJ, Yeh YH, See LC. Association of metformin with lower atrial fibrillation risk among patients with type 2 diabetes mellitus: a population-based dynamic cohort and in vitro studies. Cardiovasc Diabetol. 2014 Aug 10;13:123.

12. Liou YS, Yang FY, Chen HY, Jong GP. Antihyperglycemic drugs use and new-onset atrial fibrillation: A population-based nested case control study. PLoS One. 2018;13:e0197245.

13. Fang WT, Li HJ, Zhang H, Jiang S. The role of statin therapy in the prevention of atrial fibrillation: a meta-analysis of randomized controlled trials. Br J Clin Pharmacol. 2012;74:744-56.

14. Sai C, Li J, Yingbin X, Ruiyan M. Atorvastatin prevents postoperative atrial fibrillation for patients undergoing cardiac surgery. Hellenic J Cardiol. 2019;60:40-47.

15. Rozen G, Hosseini SM, Kaadan MI, Biton Y, Heist EK, Vangel M, Mansour MC, Ruskin JN. Emergency Department Visits for Atrial Fibrillation in the United States: Trends in Admission Rates and Economic Burden From 2007 to 2014. J Am Heart Assoc. 2018 Jul 20;7(15).

16. Tseng $\mathrm{CH}$. Metformin is associated with a lower risk of colorectal cancer in Taiwanese patients with type 2 diabetes: a retrospective cohort analysis. Diabetes Metab. 2017;43:438-45.

17. Parsons LS. Performing a 1:N case-control match on propensity score. http://www.google.com.tw/url? sa=t\&rct=j\&q=\&esrc=s\&source=web\&cd=1\&ved=0CBsQFjAAahUKEwibi7HIlcnIAhUDoJQKHVeZA9A\&url= http\%3A\%2F\%2Fwww2.sas.com\%2Fproceedings\%2Fsugi29\%2F165-29.pdf\&usg= AFQjCNFOHGWYu8E8Bn4-Bo1TUiJKtT987Q (last accessed February 22, 2020).

18. Chang L. A study of validation on comorbidity derived from claims data [Master thesis]. National Yang-Ming University; 2004. https://etd.lib.nctu.edu.tw/cgi-bin/gs32/ymgsweb.cgi/ccd=pfvwnT/fulltextstdcdr?dbid=glzDVcOUZLOa\&dbpathf= /opt/fb32/db/ym/ymcdrf/\&fuid=01\&dbna= (Accessed: February 22, 2020).

19. Tseng CH. Diabetes, metformin use, and colon cancer: A population-based cohort study in Taiwan. Eur J Endocrinol. 2012;167:409-16.

20. Austin PC, Stuart EA. Moving towards best practice when using inverse probability of treatment weighting (IPTW) using the propensity score to estimate causal treatment effects in observational studies. Stat Med. 2015;34:3661-79.

21. Austin PC. The performance of different propensity score methods for estimating marginal hazard ratios. Stat Med. 2013;32:2837-49. 
22. Kesmodel US. Information bias in epidemiological studies with a special focus on obstetrics and gynecology. Acta Obstet Gynecol Scand. 2018;97:417-423.

23. Viollet B, Guigas B, Sanz Garcia N, Leclerc J, Foretz M, Andreelli F. Cellular and molecular mechanisms of metformin: an overview. Clin Sci (Lond). 2012;122:253-70.

24. Bai F, Liu Y, Tu T, Li B, Xiao Y, Ma Y, Qin F, Xie J, Zhou S, Liu Q. Metformin regulates lipid metabolism in a canine model of atrial fibrillation through AMPK/PPAR-a/VLCAD pathway. Lipids Health Dis. 2019;18:109.

25. Packer M. Drugs that ameliorate epicardial adipose tissue inflammation may have discordant effects in heart failure with a preserved ejection fraction as compared with a reduced ejection Fraction. J Card Fail. 2019;25:986-1003.

26. Stanciu AE, Vatasescu RG, Stanciu MM, Serdarevic N, Dorobantu M. The role of pro-fibrotic biomarkers in paroxysmal and persistent atrial fibrillation. Cytokine. 2018;103:63-68.

27. Song Y, Chen Y, Li Y, Lyu X, Cui J, Cheng Y, Zhao L, Zhao G. Metformin inhibits TGF- $\beta$ 1-induced epithelial-to-mesenchymal transition-like process and stem-like properties in GBM via AKT/mTOR/ZEB1 pathway. Oncotarget. 2017;9:7023-7035.

28. Lévesque LE, Hanley JA, Kezouh A, Suissa S. Problem of immortal time bias in cohort studies: example using statins for preventing progression of diabetes. BMJ. 2010;340:b5087.

\section{Figures}

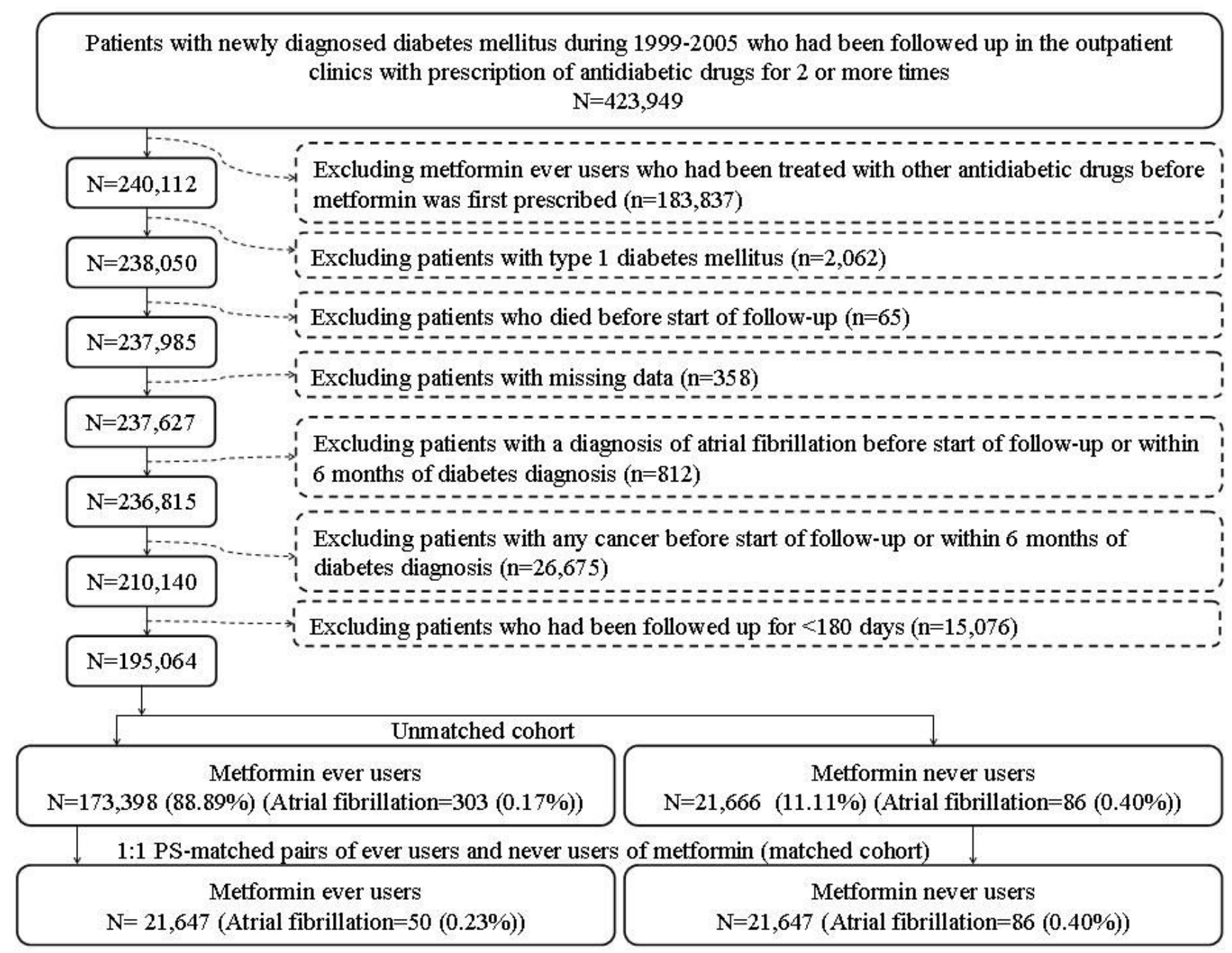

\section{Figure 1}

Flowchart showing the procedures in creating an unmatched cohort and a cohort of 1:1 propensity score-matched-pairs of metformin ever users and never users derived from the reimbursement database of the Taiwan's National Health Insurance (PS: propensity score). 


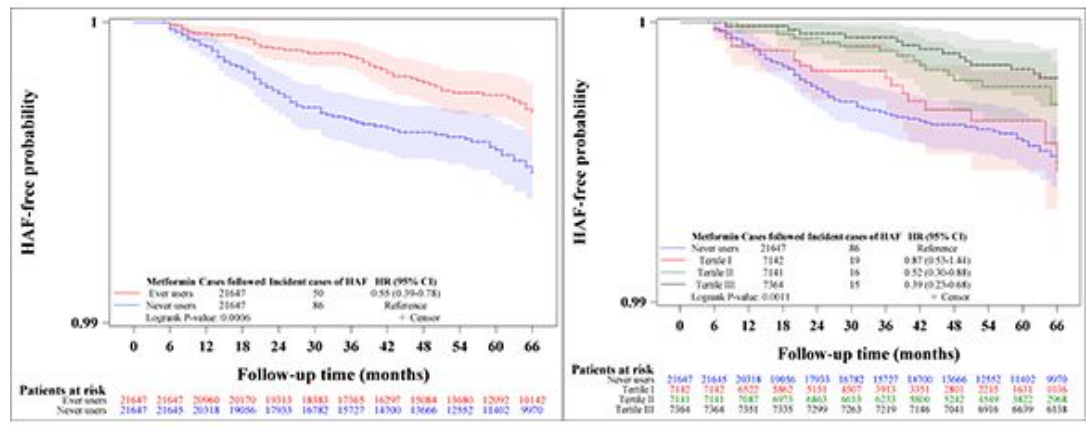

Figure 2

Kaplan-Meier curves comparing hospitalization for atrial fibrillation (HAF)-free probability in never users and ever users of metformin (Figure 2A) and in never users and users in each tertile of cumulative duration of metformin therapy (Figure $2 \mathrm{~B}$ ). The $95 \%$ confidence intervals are shown in shaded areas. HR: hazard ratio; $\mathrm{Cl}$ : confidence interval. 